\title{
Personal stake
}

\author{
Robert West ${ }^{1}$ \\ 1 University College London, University of London
}

A personal attribute that involves an outcome of value to that person arising from a process.

Curator note: This is derived from the idea of a stakeholder and is a generalisation of vested interest.

Elaboration: Value here can be positive or negative. This definition refers to actual value rather than perceived value. The process that produces the outcome will often be an event such as reporting of the outcome of a clinical trial, or delivery of a verdict in a trial, or enactment of legislation. 